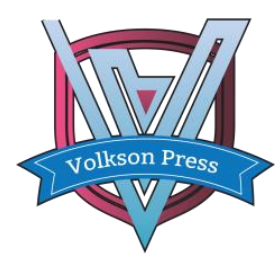

Contents List available at VOLKSON PRESS

Mechanical and Control Engineering (MCE)

DOI : http://doi.org/10.26480/wsmce.01.2017.177.180

\title{
ALGORITHM RESEARCH ON CONFLICT DETECTION AND ELIMINATION IN COLLABORATIVE WORK
}

\author{
Teng Haikun*, Li Lunbin, Cong Wanjuan \\ Computer and Information Engineering College, Heihe University Academic Road No. 1, Heihe City, China \\ *Corresponding Author Email: thk_1983@163.com
}

This is an open access article distributed under the Creative Commons Attribution License, which permits unrestricted use, distribution, and reproduction in any medium, provided the original work is properly cited

\section{ARTICLE DETAILS}

\section{Article History:}

Received 02 october 2017 Accepted 06 october 2017 Available online 11 november 2017

Keywords

Collaborative work, Conflict detection and elimination, Supply and demand of time, Heuristic rules

\section{ABSTRACT}

For cross-enterprise resource conflict problem in collaborative work plan, put forward a new kind of conflict detection and elimination of cross-enterprise project work model, based on the time of supply and demand plan collaboration conflict detection algorithm of cross-enterprise collaborative project resource conflict detection, the plan conflict resolution algorithm based on heuristic rules to eliminate conflict. Based on the results of the study project plan management prototype system is designed and developed, in the management of tasks for high-speed rail projects, for example verified, show the effectiveness of the results of the study.

\section{INTRODUCTION}

With the increasing number of cross-industry and cross-region interenterprise product management collaboration, large enterprises - crossenterprise cooperation projects have sprung up. Project planning is the core of cross-enterprise project management, and it is a means to avoid waste of resources and efficient organizations. So, the collaborative planning is also very important in cross-enterprise project management [1]. Resources are the guarantee of project implementation and the final implementation. For cross-enterprise projects of large manufacturing enterprises, complex product structure, distributed project organization, the long project execution cycle may lead to resources time overlaps, which is the key resources competition. This leads to a different plan conflict between businesses, affect the whole process of the project [2]. Constraints for cross-enterprise project task sets, we set a Net Plan with unlimited capacity constraints, important parameters such as ask schedule, key path and execution cycle of the task are determined. Based on this, considering the current resources dynamic utilization, Period of time planning for the network dynamic capabilities of statistics and contrast, Find out when the resources conflict, and the generate resource conflict related tasks, and then conflict the resolution. Finally determine the project execution plan and resource allocation $[3,4]$. This passage is aiming at the real-time resources conflict detection and elimination strategy for research.

Based on the complex product manufacturing companies High-Speed Railway production of one of the enterprises as the background in the process of cross-enterprise project planning, the collision detection algorithm based on time of supply and demand conflicts occurring in the process of plan coordination resources for real-time detection, the conflicts elimination algorithm based on heuristic rules to eliminate, finally set the project schedule and resource management.

\section{OVERVIEW OF THE ACROSS-ENTERPRISE'S COLLABORATIVE PROCESS PLANNING}

The across-enterprise's project plan occupies an important position in project management, it will directly affect the implementation of crossenterprise projects, also directly related to the operation of multiple enterprises. Consider the complexity of cross-enterprise projects; there are three stages to the collaborative process of the inter-enterprise project: Collaborative control planning stages, Equipment production schedule control stage, Collaboration control plan change phase $[5,6]$. The collaboration of cross-enterprise project planning is mainly happening in the preparation stage of collaborative control planning.

This passage is aim at the equipment requirements planning model of the assembly enterprises ( $\mathrm{ZGJ}$ ) and the collaborative enterprise equipment supply plan model (XGJ) work out the collaborative process of network planning. Based on the time parameter, critical path which related to the task, we put forward two collaborative work phases based on the collaborative conflict detection and conflict resolution, see the figure 1 for details. At first, this phase carried out the collaborative conflict detection for the equipment demand plan of assembly enterprises and the equipment supply plan of the key cooperative enterprise. When there is a non-normal supply situation, according to the corresponding conflict elimination method, then evaluates the elimination strategy according to the predefined performance evaluation index, the selected elimination strategy will be sent to the general assembly enterprise and the cooperative enterprise to carry out the plan implementation eventually [7, 8]. Resubmit the demand plan and supply plan to generate a new network plan after the elimination strategy is implemented by the general assembly enterprise and key cooperative enterprise until there is no scheduled conflict.

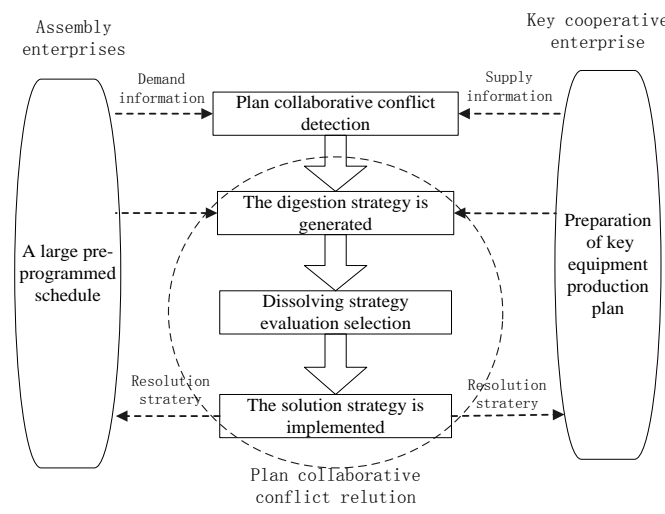

Figure 1: Planned collaborative process 


\section{CROSS-ENTERPRISE PROJECT PLAN COLLABORATIVE CONFLICT DETECTION ELIMINATION ALGORITHM}

3.2

\section{Problem description}

In the collaborative process of cross-enterprise project planning, there may be various conflicts between the equipment demand planning and supply situation. The main causes of resource conflict can be divided into: Supply and demand time, that is, the time of the task overlaps; the delay in the preorder task has an impact on the next task; the task time does not match the resource scheduling calendar. The common reason for this is tasks resource overlap the time [9]. Therefore, this passage aims at the equipment supply and demand time to carry out the collaborative conflict detection. Mainly to complete the equipment of final assembly enterprises demand plan and, collaboration enterprise supply plan test time conflict of supply and demand, according to the test results, the change of the next plan is determined.

First, select equipment requirement information and corresponding equipment supply plan. Then carry out the, scheduling conflict detection based on supply and demand time, according to different test results, the predetermined strategy is processed. The execution flow of the planned conflict detection elimination is shown in figure 2 . When the equipment is not normally supplied, the whole mission was postponed due to the failure of the equipment to arrive at the original plan. At this time, it is believed that the overall plan prepared by the general assembly enterprises and the planned production and supply plan of equipment produced by the key cooperative enterprises are planned to cooperate with the conflict.

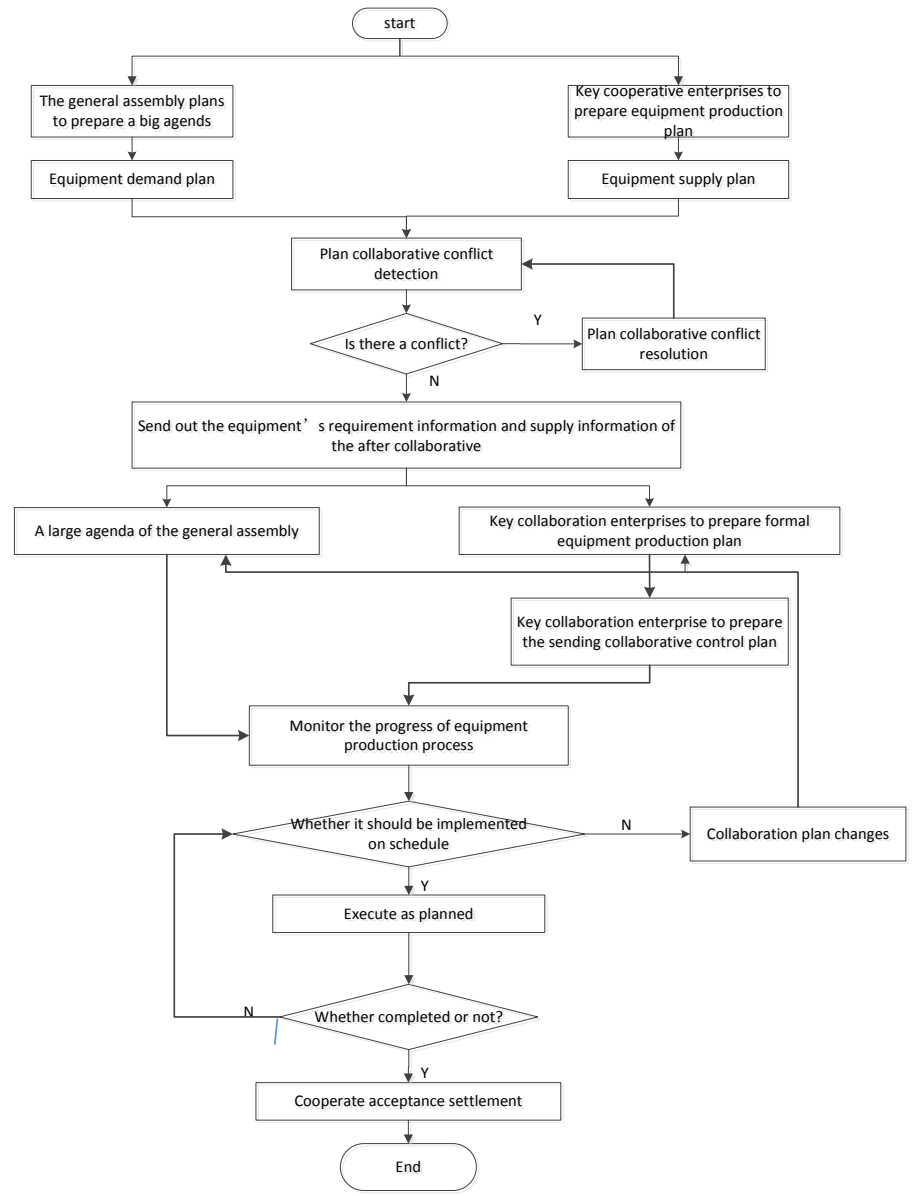

Figure 2: Plan conflict detection and elimination of the implementation process

\subsection{A collaborative conflict detection algorithm based on} supply and demand time

According to the supply time of key cooperative enterprises and the relationship between the earliest delivery time and the latest delivery time required by the general assembly enterprise, the conflict detection algorithm based on the time of supply and demand is proposed during the conflicts in process of network planning. The algorithm is determined by the time of supply and demand: Supply mode, type of conflict and other relevant information; Conflicting supply constraints information for subsequent collaboration devices; The impact of the conflict on the production plan. Our passage divides the conflict type into four categories: early supply, normal supply, delayed supply direct conflict and indirect conflict. The conflict detection algorithm based on supply and demand time is described as follows.

\section{Input : ZGJ $\mathrm{XGJ} 、$ NetPlan}

Output : Test result type

Specific description of algorithm:

Conflict detection classification and arrangement process

Acquiring ZGJ's equipment number and arrange the earliest demand time according to the general assembly enterprise from morning to evening $G_{-}$Set $=\left\{G_{1}, G_{2}, \cdots G_{n}\right\}$

The initialization constraint is empty

for 1 to $\mid G_{-}$Set $\mid / /$Cycle test

If The supply time is less than the earliest demand time early delivery

$$
\text { Maximum deviation }
$$

$C M_{i}$

=Earliest delivery time-Delivery time

Add early supply information to the collection of early supply equipment ;

Else if supply normally

Add normal supply information to the collection of normal supply equipment;

supply

Else Direct conflict arising from the delay of

Maximum deviation $C M_{i}=$ Delivery time-Latest delivery time;

Deeply travel network diagram to find ${ }^{{ }_{i}}$ node's children's node; //

SON=Depth_First_Search $\left(\right.$ NetPlan,$G_{i}$ )

for $G_{i}$ 's every children's node $G_{k} \in S O N$

If $G_{k} \in\left\{G_{i+1}, \cdots, G_{n}\right\} \quad / /$ Determine whether the

following collaboration tasks are affected

add $G_{k}$ to $Z G J_{i} \cdot C R_{i}$; $\quad$ //A collection of subsequent collaboration devices affected by a direct conflict of $G_{i}$ equipment delay ;

Add $G_{i}$ to $Z G J_{k} \cdot G C R_{k} ; \quad / / G C R$ Represents a set of conflicting constraints for devices

Add delay supply information to a direct conflict of supply;

delay of supply

Else Indirect conflict arising from the

indirect conflict collection;\}

Add delay supply information to the

3.4 Scheduling conflict elimination algorithm based on heuristic rules

For early delivery situation, this passage through the key collaboration enterprises and the general assembly enterprises to bear the additional costs to realize benefit-sharing, risk-sharing between the across enterprise organizations. The goal is to achieve win-win results. This passage mainly studies the delay supply situation. In allusion to the direct conflict arising from the delay of supply , the conflict elimination algorithm based on heuristic rules is adopted to deal with it ; for the indirect conflict of the supply of equipment to be delayed for the previous task, use a new conflict detection algorithm to detect the conflict detection algorithm after the direct conflict is eliminated, In the end, indirect conflict is transformed into general supply situation or direct conflict situation. The proposed conflict resolution algorithm based on heuristic rules is described as follows.

input : A collection of planned conflicts resulting from an extension of supply 、ZGJ、XGJ 、NetPlan 、Free relaxation time FL 、 Maximum deviation of equipment $C M_{i}$

output : Plan conflict resolution policy collection $C C L$

Description of algorithm :

Step1 : Initialize the active queue $Q$ and Conflict elimination strategy collection $C C L$;

Step : According to the project scheduling rule respectively and task 
scheduling rule, Sorting data items $P R_{i}$ for collection $C_{-} 3$ of test results and task decomposition $W B S_{i}$ by radix , The processing priority is inserted in the queue $Q$ from the previous to the following ;

Step3 : Judge whether $Q$ is empty •If it's empty, the algorithm ends ; else, Remove the first element ${ }_{i j}$ from the $Q$, do Step4-9, until the queue is empty

Step4 : Judge whether $a_{i j}$ is key activity. If it is, turn to Step8 ; Otherwise it will continue ;

Step5 : Judge $a_{i j}$ whether meet limited resource constraints for $S_{i j}$ as starting work period move $\min \left\{C M_{i}, F L_{i j}\right\}$
scheduled start time
units to the right $\circ$ If it meets, turn to Step7 ; Otherwise it will continue \[ S_{i j} \text { as starting work period move } \min \left\{C M_{i}, F L_{i j}\right\} \]
scheduled start time
units to the right $\circ$ If it meets, turn to Step7 ; Otherwise it will continue Step6 : Calculate the maximum time that $a_{i j}$ can move to the right under the constraints of existing resources. Start with $S_{i j}$, The task period moves t units to the right, $C M_{i}$ updated to $C M_{i}-t$, put $a_{i j}$ to the queue $Q$, turn to Step3;

Step7 : Determine the total cost of adjusting for non-critical activities $D_{i j}^{1}$ $L_{i j}$ cooperative enterprise, turn to Step10 ;

Step8 : For key activity $a_{i j}$, the following three schemes will be adopted to generate the conflict elimination strategy

Scheme 1 : The conflict can be eliminated by the general assembly alone. The coordination center calls the key task time dynamic scheduling algorithm of the general assembly enterprise to generate the corresponding elimination strategy ;

Scheme 2 : The conflict can be eliminated by a collaborative enterprise alone. The coordination center calls on the project period compression algorithm based on network plan to generate the corresponding elimination strategy ;

Scheme 3 : The two sides should work together to resolve the conflict 。 At this time, the two parties respectively bear the conflict elimination time respectivelyt $1, \mathrm{t} 2,\{(\mathrm{t} 1, \mathrm{t} 2) \mid \mathrm{t} 1+\mathrm{t} 2=\mathrm{CMi}, \mathrm{t} 1>0, \mathrm{t} 2>0\} \quad, \quad$ The coordination center calls corresponding elimination algorithms to generate corresponding policies ;

Step9 : The coordination center evaluates the above generated strategy using performance evaluation index, Choose the best conflict resolution strategy $L_{i j}$ hand out the general assembly enterprise and the collaboration enterprise to implement If there is a consensus, then turn to Step10 。 If an agreement is not reached, then the suboptimal elimination strategy is selected until the consensus or elimination strategy is blank - If the elimination strategy is empty, The coordination center and the two parties negotiate the duration of the project and the corresponding coordination strategy, agree on the two parties to ensure the minimum duration of the delay, turn to Step10.

Step10 : The expenses incurred by both parties shall be calculated according to the apportionment mechanism agreed in advance by both parties, generate the final conflict elimination strategy, the two sides collaborate on this, the algorithm ends.

\section{SYSTEM INSTANCE VERIFICATION}

According to the cross-enterprise project plan collaboration plan conflict detection method and research on management techniques, This passage is based on the high-speed rail manufacturing enterprises, established a project resource management prototype system , this system included Planning compile management, Planning schedule management, Collaboration plan change management - Plan collaborative conflict detection and Plan collaborative conflict elimination, and offered Project network planning - resource conflict detection during execution expression of test results and conflict resolution based on planned dynamic adjustment. The coordination center will conduct coordinated conflict detection based on supply and demand time based on the equipment supply plan submitted by the total installed enterprises and the equipment supply plan submitted by the key cooperative enterprise. The results of the planned collaborative conflict detection are shown in figure 3.

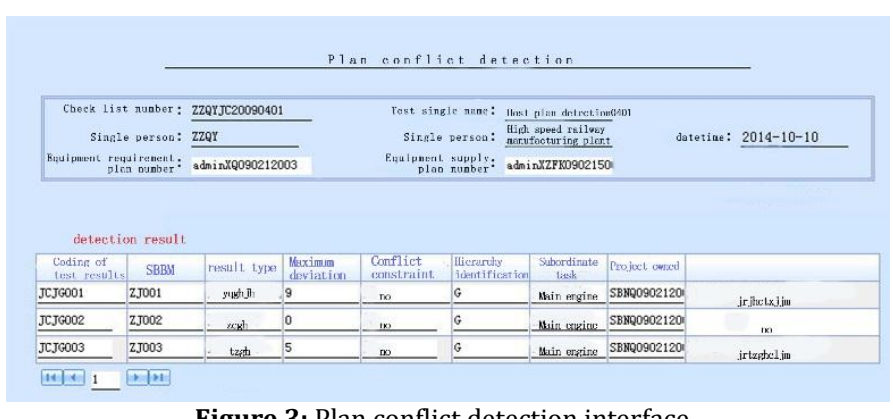

Figure 3: Plan conflict detection interface

According to different types of test results, different treatments are performed. When the equipment is delayed and the planned conflict is caused, The method of planning conflict elimination based on heuristic rules is used, the resulting interface is shown in figure 4.The coordination center reviews the generated planned conflict resolution strategies based on predefined performance reviews, and based on the results of the comparison, select the optimal elimination strategy to allocate the conflict elimination strategy for the assembly enterprises and the cooperative enterprises.

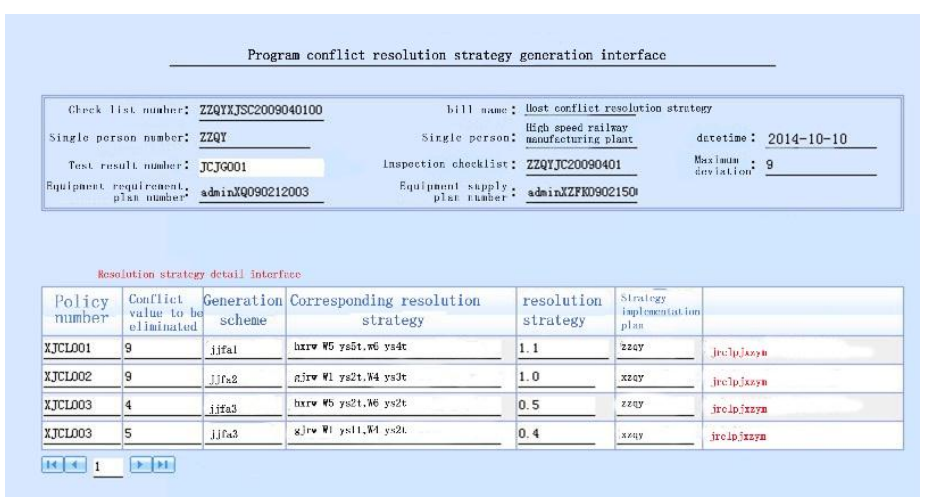

Figure 4: Plan conflict elimination strategy generation interface

Example verification shows that, the collaborative conflict detection algorithm can effectively identify resource conflicts in project tasks, the expression of multiple views is intuitive and explicit. The system is very effective in managing resource planning conflict information.

\section{CONCLUSION}

Based on the high-speed rail project tasks as the background, through the plan collaboration conflict detection algorithm based on supply and demand of time and its elimination strategy based on heuristic rules in the application of cross-enterprise project research, solved in across the enterprise project management system of related issues, greatly improving the efficiency of the operation of the joint between enterprises established and verified through an example of the feasibility of the project resource management prototype system, to solve the problem of resource management of cross-enterprise collaborative project provides an effective solution. Plan for cross-enterprise collaborative project resources conflict problem in the theory and method of research still need to continue to a large number of research and exploration, to meet the actual needs of large project management in our country.

\section{REFERENCES}

[1] Zhenggeng, Q. 2012. Research on conflict resolution mechanism in collaborative work [J]. Computing technology and automation, 31 (3), 104-108.

[2] Qiaolihong, Wangchao. 2008. Resource conflict detection and management in multi-level collaborative project execution [J]. Journal of Beijing university of aeronautics and astronautics, 34 (11), 1266-1271. 
[3] Zheng, Y., Wang, J., Xue, J. 2007. Distributed Cooperative Planning and Scheduling for Disaster Management [J]. IEEE International Conference.

[4] Heting, Wudongdong, Xuhanchuan. 2008. Research on crossenterprise collaborative planning based on PM/ERP integration model [J]. Computer engineering and design.

[5] Ming, L., MaShiHua. 2003. Dynamic alliance project management new mode [M]. Electronic industry press.

[6] Li, M.X. 2010. Collaborative design support environment and conflict elimination theory and method [M]. Nanjing: southeast university press.
[7] Li, Z. 2010. Study on the method of conflict elimination in the collaborative development of sewing equipment [J]. Journal of engineering design, 17 (2), 188-190.

[8] Wangliping, Bo, J., Yue, Q.F. 2010. A collaborative design conflict resolution method based on multi-objective evolutionary algorithm [J]. Computer integrated manufacturing system, 16 (9), 174-176.

[9] Yu, Y., Lei, W.X., Qiang, Z. 2011. Multi-principal conflict coordination in collaborative product innovation design optimization [J]. Computer integrated manufacturing system, 17 (1), 255-258 